\title{
Ultrahigh temperature-sensitive silicon MZI with titania cladding
}

\author{
Jong-Moo Lee ${ }^{1,2 *}$ \\ ${ }^{1}$ Electronics and Telecommunications Research Institute, Daejeon, South Korea, ${ }^{2}$ School of Advanced Device Technology, \\ University of Science and Technology, Daejeon, South Korea
}

We present a possibility of intensifying temperature sensitivity of a silicon Mach-Zehnder interferometer (MZI) by using a highly negative thermo-optic property of titania $\left(\mathrm{TiO}_{2}\right)$. Temperature sensitivity of an asymmetric silicon MZI with a titania cladding is experimentally measured from +18 to $-340 \mathrm{pm} /{ }^{\circ} \mathrm{C}$ depending on design parameters of $\mathrm{MZI}$.

Keywords: silicon, photonics, temperature, sensor, titania

\section{Introduction}

There have been many efforts to adjust temperature-dependent wavelength shift (TDWS) of

OPEN ACCESS

Edited by:

Jifeng Liu,

Thayer School of Engineering, USA

Reviewed by:

Raul J. Martin-Palma,

Universidad Autonoma de Madrid,

Spain

Venu Gopal Achanta,

Tata Institute of Fundamental

Research, India

*Correspondence:

Jong-Moo Lee

Electronics and Telecommunications Research Institute, 161 Gajong-dong, Yusong-gu, Daejeon 305-350, Korea jongmoo/@etri.re.kr

Specialty section:

This article was submitted to Optics and Photonics, a section of the journal Frontiers in Materials

Received: 30 January 2015 Accepted: 08 April 2015

Published: 05 May 2015

Citation:

Lee J-M (2015) Ultrahigh

temperature-sensitive silicon MZI with titania cladding.

Front. Mat. 2:36.

doi: 10.3389/fmats.2015.00036 a photonic waveguide device using a cladding material with a negative thermo-optic coefficient (TOC) differently from a core material with a positive TOC (Kokubun et al., 1998; Lee et al., 2007, 2008; Alipour et al., 2010; Guha et al., 2013; Bovington et al., 2014; Lee, 2014). Polymers have been popularly used as the cladding material with a negative TOC (Kokubun et al., 1998; Lee et al., 2007, 2008), and titania $\left(\mathrm{TiO}_{2}\right)$ is recently attracting attention with a highly negative TOC (Alipour et al., 2010; Guha et al., 2013; Bovington et al., 2014; Lee, 2014) and its merit of complementary-metal-oxide-semiconductor (CMOS) compatibility in fabrication when it is used for a silicon photonic waveguide device. Silicon has a very high TOC of $1.8 \times 10^{-4} /{ }^{\circ} \mathrm{C}$ and there have been many efforts to reduce the high TDWS of silicon photonic devices such as a ring resonator by using polymer (Kokubun et al., 1998; Lee et al., 2007, 2008) or titania cladding (Alipour et al., 2010; Guha et al., 2013; Lee, 2014) with a highly negative TOC.

In case of silicon photonic Mach-Zehnder interferometer (MZI), there were reports showing the way to reduce the TDWS of MZI without using a cladding with a negative TOC (Uenuma and Moooka, 2009; Guha et al., 2010; Dwivedi et al., 2013). TDWS of silicon MZI was shown to be reduced by using different widths of waveguide (Uenuma and Moooka, 2009; Guha et al., 2010) or by using different polarization (Dwivedi et al., 2013) in each of the MZI arm, respectively. The difference in each of the MZI arm can induce a different temperature-dependent phase change for the each arm, resulting reduction in TDWS of MZI.

The previous efforts of silicon photonic devices using a negative thermo-optic cladding have been focused on reducing the TDWS, but there have been demands also on high TDWS in such applications of low power temperature tuning (Masood et al., 2013) and integrated-photonic temperature sensors (Irace and Breglio, 2003; Kim et al., 2010; Deng et al., 2014). So, it would also be attractive if there is a method to intensify the TDWS of the silicon device by using a cladding material such as titania with a very high TOC. There have been MZIs using titania for chemical sensing (Qi et al., 2002; Celo et al., 2009), but no reports on temperature sensors using titania cladding to the best of our knowledge.

In this regard, here, we combine the method used to in reducing TDWS of silicon MZI with different dimension for each arm and the method of adding titania cladding on the silicon MZI to show the possibility of ultrahigh temperature-sensitive silicon MZI. 


\section{Experiment and Results}

\section{Design and Fabrication}

Temperature dependence of a silicon MZI can be adjusted by asymmetric geometry of two waveguide arms with different effective refractive indexes induced by different cross-sectional dimension as in reference Uenuma and Moooka (2009) and Guha et al. (2010). Silicon MZIs in this experiment are designed with variations in the length of MZI arm and cross-sectional dimension of each MZI arm as in Figure 1. Figure 1A shows AsyL, which is for asymmetry in the length of each MZI arm of $80 \mu \mathrm{m}, \mathrm{L}$ for the common length of MZI arm, which is varied from 110 to $360 \mu \mathrm{m}$, w0 for the common width of waveguide core, which is $450 \mathrm{~nm}$, w1 for the cross-sectional dimension of a waveguide core which is $1350 \mathrm{~nm}$ and shaped as a rib waveguide shown in Figure 1B or $450 \mathrm{~nm}$ and shaped as a channel waveguide for a comparison, and w2 for the cross-sectional dimension of a waveguide core which is 350 or $450 \mathrm{~nm}$ for a comparison. Figures 1B,C show the crosssectional structure of the waveguide with a silica cladding and a titania cladding, respectively. The rib waveguide is formed by shallow etch of $70 \mathrm{~nm}$ for the width $\mathrm{w} 1$, and there are tapers at the both ends of the rib waveguide for an adiabatic transfer to the channel waveguide with the width of w0.

Figure 2 shows a microscopic image and scanning electron microscope (SEM) image of the fabricated MZIs. There are many variations for the length, $\mathrm{L}$, in design, but we limit our discussion here to the two extreme case of L, 110 and $360 \mu \mathrm{m}$. Silicon waveguide core with the width of $450 \mathrm{~nm}$ was patterned by DUV lithography on a silicon-on-insulator (SOI) wafer with a 220 -nm thick silicon layer on a $2-\mu \mathrm{m}$ thick buried oxide (BOX) layer. The fabrication of the devices except a deposition of titania cladding were processed using a standard CMOS fabrication process through ePIXfab. There were two types of fabricated device: one with a silica $\left(\mathrm{SiO}_{2}\right)$ cladding and the other without an upper

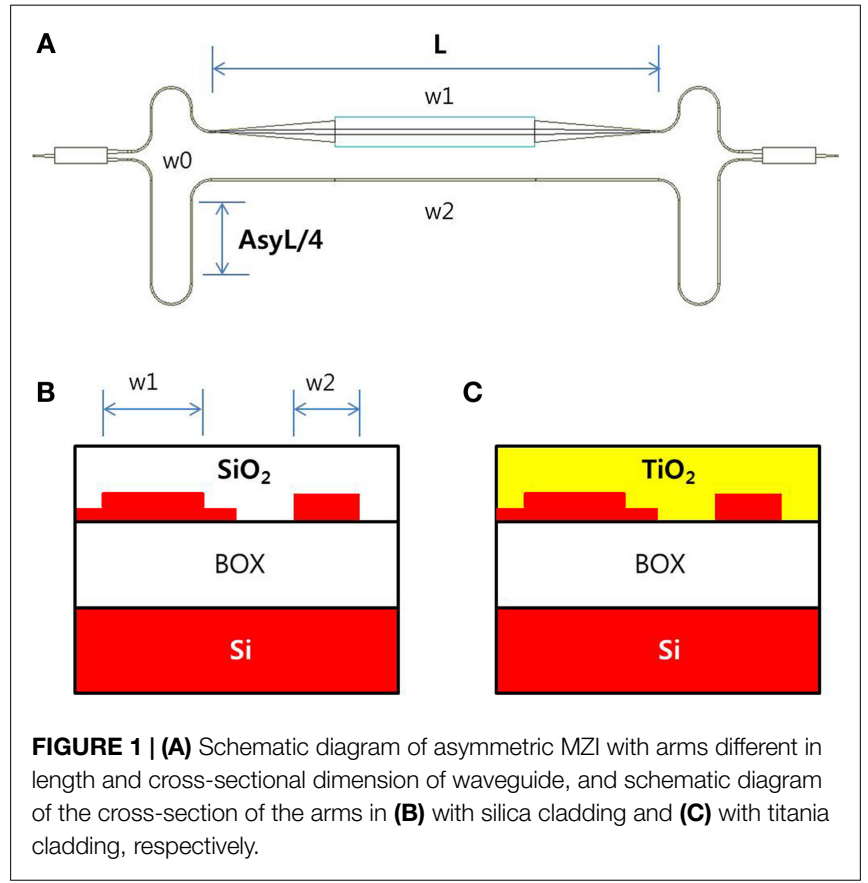

cladding. We deposited $400 \mathrm{~nm}$ thickness of titania cladding on the fabricated device without an upper cladding, using electronbeam evaporation. The initial vacuum level of the electron-beam evaporation was $5 \times 10^{-7}$ Torr, and it was kept at $8 \times 10^{-5}$ Torr with $\mathrm{O}_{2}$ during the deposition. The temperature of a plate holding SOI chip was maintained at $150^{\circ} \mathrm{C}$ during the evaporation, and the speed of deposition was about $3 \AA /$ s. The refractive index of titania was measured using ellipsometry as 2.13 at $1550 \mathrm{~nm}$.

\section{Measured Results}

One pair of single-mode fibers is coupled to the silicon devices for measurement through grating couplers which are with 630$\mathrm{nm}$ pitch and $70 \mathrm{~nm}$ depth of the shallow etch. Figure 3 shows normalized transmission spectra of a silicon MZI with a silica

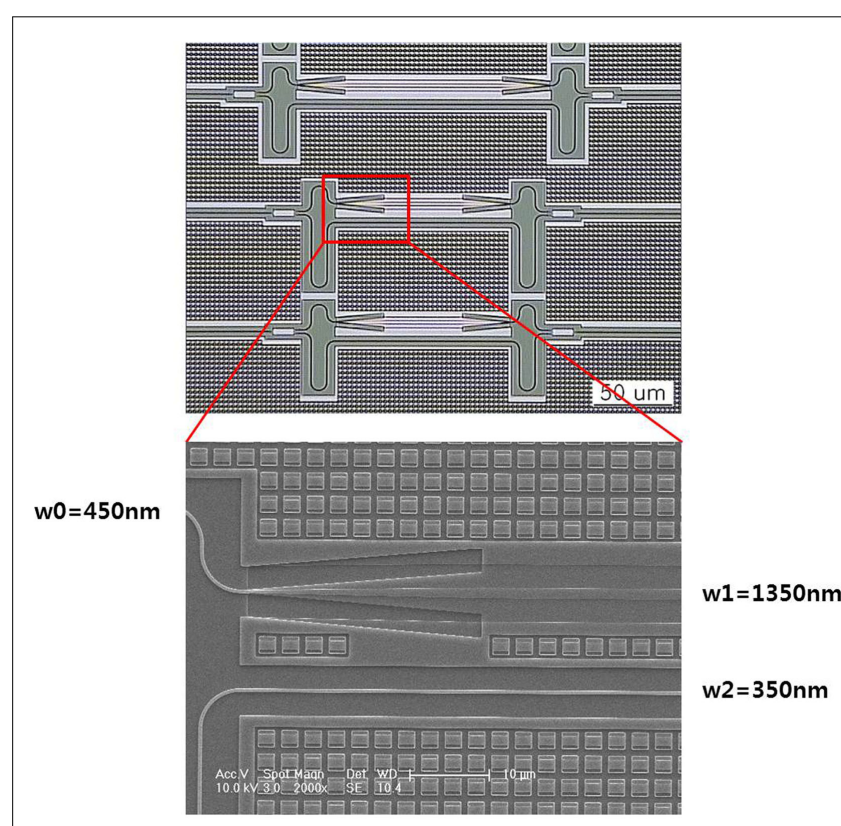

FIGURE 2 | A microscope image and a SEM image of silicon MZIs fabricated in this experiment

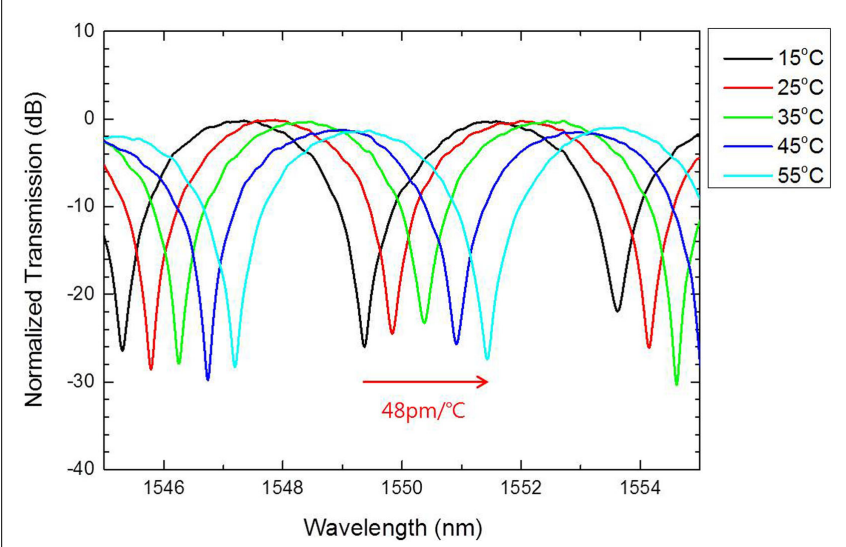

FIGURE 3 | Normalized transmission spectra of MZI with silica cladding as the temperature varied from 15 to $55^{\circ} \mathrm{C}$ when $\mathrm{L}=360 \mu \mathrm{m}$, $w 1=1350 \mathrm{~nm}$, and $w 2=350 \mathrm{~nm}$. 
cladding when the temperature varied from 15 to $55^{\circ} \mathrm{C}$. The main design parameters of the silicon MZI are $360 \mu \mathrm{m}$ for $\mathrm{L}$, $1350 \mathrm{~nm}$ for $\mathrm{w} 1$, and $350 \mathrm{~nm}$ for $\mathrm{w} 2$. Figure 3 shows TDWS of the silicon MZI is $+48 \mathrm{~m} /{ }^{\circ} \mathrm{C}$, which was reduced from $+74 \mathrm{pm} /{ }^{\circ} \mathrm{C}$ of a ring resonator included in the same chip for a comparison. The normalized transmissions can be regarded as the insertion loss of the silicon MZI, because they were calculated by subtracting the amount of fiber-to-fiber transmission of a straight silicon waveguide from the amount of fiber-to-fiber transmission of the silicon MZI device. Figure 3 shows that the insertion loss through the silicon MZI is negligibly small.

Figure 4 shows normalized transmission spectra of a silicon MZI, whose design is the same as in Figure 3 but with a titania cladding instead of the silica cladding, when the temperature varied from 25 to $35^{\circ} \mathrm{C}$. Figure 4 shows TDWS of the silicon MZI is intensified with opposite sign by the titania cladding as high as $-340 \mathrm{pm} /{ }^{\circ} \mathrm{C}$, which is about seven times bigger than the TDWS of the same design of MZI with a silica cladding and five times bigger than the TDWS of the ring resonator with a silica cladding.

Figure 5 shows the relative wavelength shift of various MZIs with silica or titania cladding in this experiment compared to the TDWS of the ring resonator with the silica cladding. The radius of the ring resonator with the silica cladding was $5 \mu \mathrm{m}$ and TDWS of the ring resonator was measured at $+74 \mathrm{pm} /{ }^{\circ} \mathrm{C}$ as in Figure 6. TDWS of a silicon MZI with the same cross-section dimension of $450 \mathrm{~nm}$ and titania cladding was measured as $+18 \mathrm{pm} /{ }^{\circ} \mathrm{C}$ as in Figure 5. TDWS of another titania-covered silicon MZI with $1350 \mathrm{~nm}$ for $\mathrm{w} 1,450 \mathrm{~nm}$ for $\mathrm{w} 2$, and $110 \mu \mathrm{m}$ for $\mathrm{L}$ was measured as $-70 \mathrm{pm} /{ }^{\circ} \mathrm{C}$ as in Figure 5.

\section{Discussion}

The experimental results show that we can adjust TDWS of the titania-covered silicon MZIs with proper design and can intensify the temperature sensitivity highly enough to be useful in applications requiring an ultrahigh temperature sensitivity such as thermo-optic tuning devices or photonic temperature sensors.

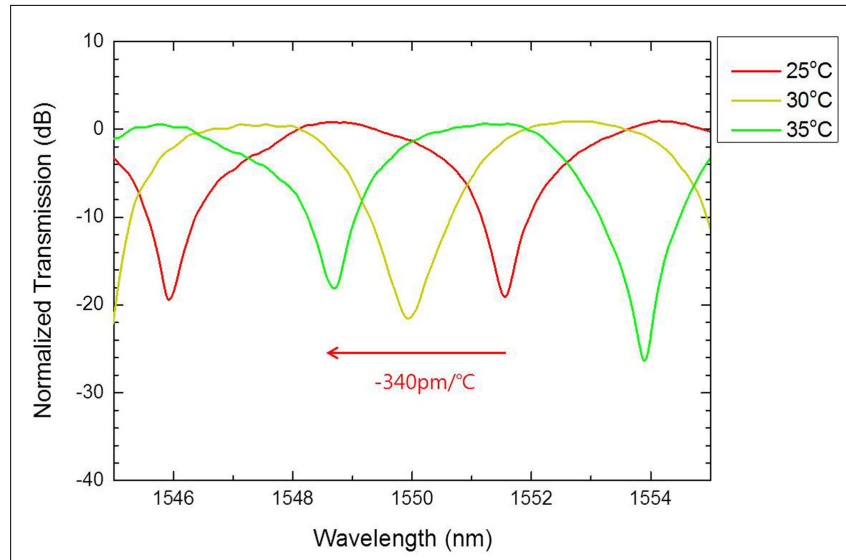

FIGURE 4 | Normalized transmission spectra of MZI with titania cladding as the temperature varied from 25 to $35^{\circ} \mathrm{C}$ when $\mathrm{L}=360 \mu \mathrm{m}$, $w 1=1350 \mathrm{~nm}$, and $w 2=350 \mathrm{~nm}$.
The input and output fibers are coupled to the silicon waveguide through grating couplers with the pitch of $630 \mathrm{~nm}$. The fiber was coupled at the vertical angle of $10^{\circ}$ for the waveguide with silica cladding and $15^{\circ}$ for the waveguide with titania cladding. There was not a big difference in the coupling loss of the grating couplers for the silica cladding and titania cladding. It was about $5 \mathrm{~dB} /$ facet for the silica cladding and $5.5 \mathrm{~dB} /$ facet for the titania cladding. The slightly excessive loss of the grating coupler in case of titania cladding is expected to be reduced by optimizing the design of gratings or the thickness of titania if it is required. The normalized transmission in Figures $\mathbf{3}$ and $\mathbf{4}$ were calculated by subtracting the amount of fiber-to-fiber transmission of a straight silicon waveguide from the amount of fiber-to-fiber transmission of the silicon MZI device for each case of silica cladding and titania cladding, respectively. So, the normalized transmission spectra show the insertion loss of MZI compared to a straight waveguide.

The refractive index of titania cladding was measured using ellipsometry as 2.13 at $1550 \mathrm{~nm}$, and TOC of titania film was
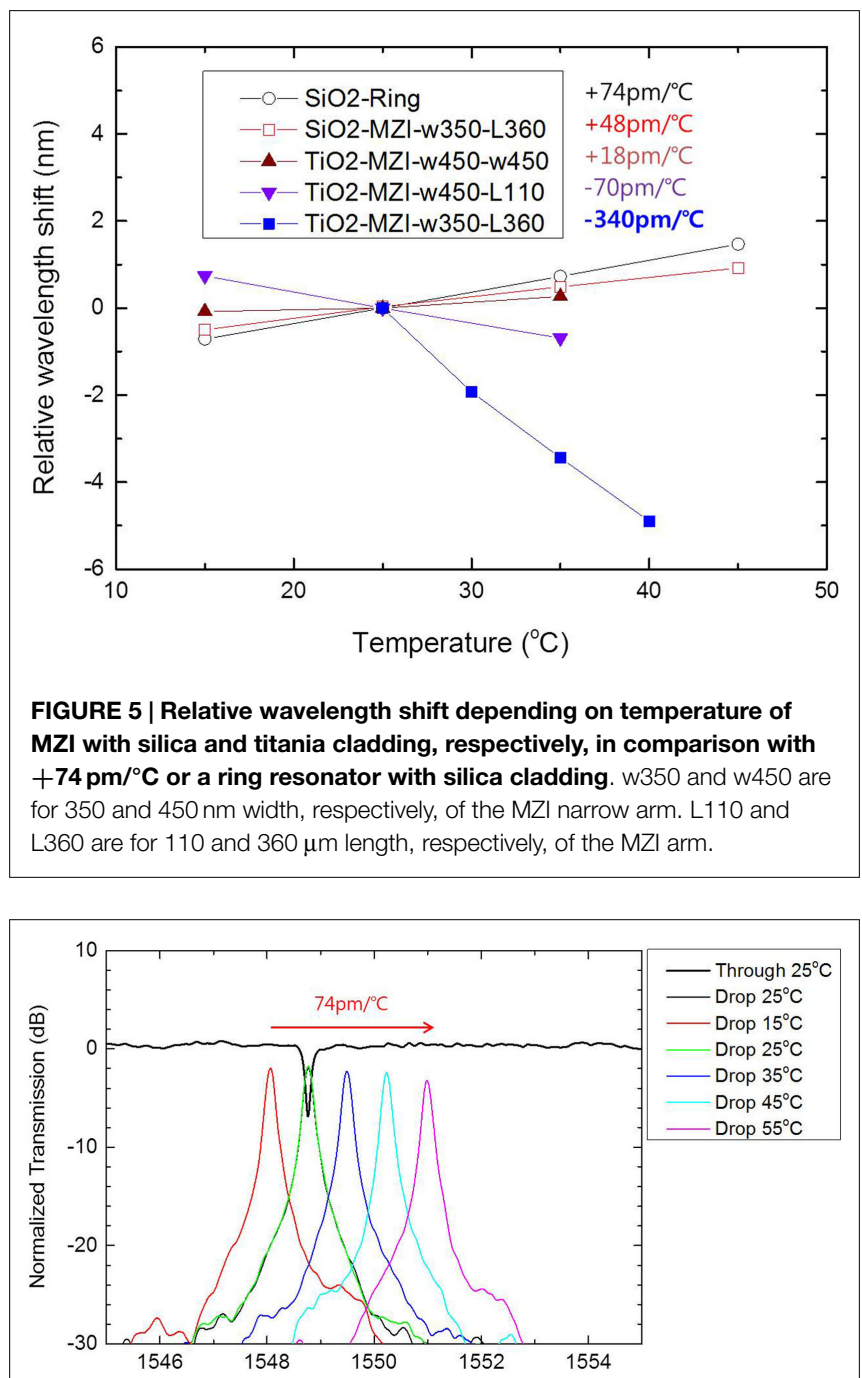

FIGURE 6 | Normalized transmission spectra of the ring resonator with silica cladding mentioned in Figure 5 , as the temperature varied from 15 to $55^{\circ} \mathrm{C}$. 
not directly measured but estimated from -5 to $-7 \times 10^{-4} /{ }^{\circ} \mathrm{C}$ by the measured TDWS of a ring resonator with titania cladding as in reference Lee (2014). The absolute value of TOC of titania is several times higher than TOC of silicon or polymer, and that is the reason we used it as highly negative thermo-optic cladding in this experiment. The reason for the highly negative TOC of the titania cladding and the variation of TOC is not fully understood yet, and finding the reason remains for our future research.

\section{Conclusion}

We experimentally showed that TDWS of a silicon MZI can be reduced or intensified by proper design of the width and length of arms of MZI when it is used with a highly negative

\section{References}

Alipour, P., Atabaki, A. H., Eftekhar, A. A., and Adibi, A. (2010). "Athermal performance in titania-clad microring resonators on SOI," in OSA Technical Digest. Integrated Photonics Research, Silicon and Nanophotonics and Photonics in Switching, Paper IMC6; 2010 Jul 25-28, (Monterey, CA: Optical Society of America).

Bovington, J., Wu, R., Cheng, K. T., and Bowers, J. E. (2014). Thermal stress implications in athermal $\mathrm{TiO} 2$ waveguides on a silicon substrate. Opt. Express 21, 661-666. doi:10.1364/OE.22.000661

Celo, D., Post, E., Summers, M., Smy, T., Brett, M. J., and Albert, J. (2009). Interferometric sensing platform with dielectric nanostructured thin films. Opt. Express 17, 6655-6664. doi:10.1364/OE.17.006655

Deng, Q. Z., Li, X. B., Chen, R. B., and Zhou, Z. (2014). "Low-cost silicon photonic temperature sensor using broadband light source," in IEEE 11th International Conference, Group IV Photonics (GFP), WP23; 2014 Aug 27-29, (Paris).

Dwivedi, S., D'heer, H., and Bogaerts, W. (2013). A compact all-silicon temperature insensitive filter for WDM and bio-sensing applications. IEEE Photonics Technol. Lett. 25, 2167-2170. doi:10.1109/LPT.2013.2282715

Guha, B., Cardenas, J., and Lipson, M. (2013). Athermal silicon microring resonators with titanium oxide cladding. Opt. Express 21, 26557-26563. doi:10. 1364/OE.21.026557

Guha, B., Gondarenko, A., and Lipson, M. (2010). Minimizing temperature sensitivity of silicon Mach-Zehnder interferometers. Opt. Express 18, 1879-1887. doi:10.1364/OE.18.001879

Irace, A., and Breglio, G. (2003). All-silicon optical temperature sensor based on multi-mode interference. Opt. Express 11, 2807-2812. doi:10.1364/OE.11. 002807

Kim, G. D., Lee, H. S., Park, C. H., Lee, S. S., Lim, B. T., Bae, H. K., et al. (2010). Silicon photonic temperature sensor employing a ring resonator manufactured using a standard CMOS process. Opt. Express 18, 22215-22221. doi:10.1364/OE. 18.022215 thermo-optic titania cladding. We experimentally showed temperature sensitivity of an asymmetric silicon MZI with a titania cladding could be adjusted from +18 to $-340 \mathrm{pm} /{ }^{\circ} \mathrm{C}$ depending on design parameters such as the width and length of MZI. We believe these results show the possibility of ultrahigh temperaturesensitive silicon MZI for new applications requiring ultrahigh temperature sensitivity such as thermo-optic tuning devices or photonic temperature sensors.

\section{Acknowledgments}

We would like to thank ePIXfab (www.epixfab.eu) for the fabrication of SOI waveguide before our deposition of titania cladding. This work was supported by Korean IT R\&D program MOTIE [N019800001] and [10044735].

Kokubun, Y., Yoneda, S., and Matsuura, S. (1998). Temperature-independent optical filter at $1.55 \mu \mathrm{m}$ wavelength using a silica-based athermal wavelength. Electron. Lett. 34, 367-369. doi:10.1049/el:19980245

Lee, J. M. (2014). Influence of titania cladding on SOI grating coupler and $5 \mu \mathrm{m}-$ radius ring resonator. Opt. Commun. 338, 101-105. doi:10.1016/j.optcom.2014 10.039

Lee, J. M., Kim, D. J., Ahn, H. Y., Park, S. H., and Kim, G. O. (2007). Temperature dependence of silicon nanophotonic ring resonator with a polymeric overlayer. J. Lightwave Technol. 25, 2236-2243. doi:10.1109/JLT.2007.899792

Lee, J. M., Kim, D. J., Kim, G. H., Kwon, O. K., Kim, K. J., and Kim, G. O. (2008) Controlling temperature dependence of silicon waveguide using slot structure. Opt. Express 16, 1645-1652. doi:10.1364/OE.16.001645

Masood, A., Pantouvakiy, M., Lepagey, G., Verheyeny, P., Campenhouty, J. V., Absily, P., et al. (2013). "Comparison of heater architectures for thermal control of silicon photonic circuits," in IEEE 10th International Conference, Group IV Photonics (GFP), ThC2; 2013 Aug 28-30, (Seoul).

Qi, Z., Matzuda, N., Itoh, K., Murabayashi, M., and Labers, C. R. (2002). A design for improving the sensitivity of Mach-Zehnder interferometer to chemical and biological measurands. Sens Actuators B Chem 81, 254-258. doi:10.1016/ S0925-4005(01)00960- 1

Uenuma, M., and Moooka, T. (2009). Temperature-independent silicon waveguide optical filter. Opt. Lett. 34, 599-601. doi:10.1364/OL.34.000599

Conflict of Interest Statement: The author declares that the research was conducted in the absence of any commercial or financial relationships that could be construed as a potential conflict of interest.

Copyright (C) 2015 Lee. This is an open-access article distributed under the terms of the Creative Commons Attribution License (CC BY). The use, distribution or reproduction in other forums is permitted, provided the original author(s) or licensor are credited and that the original publication in this journal is cited, in accordance with accepted academic practice. No use, distribution or reproduction is permitted which does not comply with these terms. 